\title{
A Searchable Encryption Scheme in a Multi-user Setting for Cloud Storage
}

\author{
Qingqing Gan $^{1}$ and Xiaoming Wang ${ }^{2 *}$ \\ ${ }^{1,2}$ Department of Computer Science, Jinan University, Guangzhou, China, 510632 \\ ${ }^{1}$ gan_qingqing@foxmail.com, ${ }^{2}$ twxm@jnu.edu.cn
}

\begin{abstract}
To support encrypted keyword queries on encrypted data and to access data in a multi-user setting for cloud storage, a searchable encryption scheme is proposed by combining Chinese Remainder Theorem with Public Encryption with Keyword Search. The proposed scheme enables data owners to outsource their encrypted data to the storage of cloud servers for a number of users, who are given the ability to generate valid queries and to access data without leaking any information. More importantly, the proposed scheme does not rely on shared keys to realize multi-user searchable encryption and user dynamics. Each authorized user in the proposed scheme has a unique key and can perform encrypted keyword queries on encrypted data as well as access data without knowledge of the keys of other users, such that when some users are added or removed, other users are unaffected. We prove that the proposed scheme is secure under Bilinear Diffie-Hellman and Hash Diffie-Hellman assumptions. The performance analysis shows that the proposed scheme has lower communication and storage overheads for both cloud servers and users than other existing schemes. In addition, the proposed scheme possesses features similar to that of most existing schemes in a single-user setting and does not downgrade because of supporting multiple users.
\end{abstract}

Keywords: outsourced data; authenticated data structure; data integrity; constant complexity

\section{Introduction}

With the development of cloud computing, the demand for data storage outsourcing has increased dramatically in recent years. However, a significant barrier to the adoption of outsourcing data is that data owners are concerned about confidential data leakage and loss of privacy because cloud service providers are not to be fully trusted.

A basic solution to this problem is that sensitive data have to be encrypted before outsourcing. This solution can protect the outsourcing data from outside attackers and cloud service providers, but searching encrypted data becomes difficult.

Numerous studies have been conducted to address this problem. However, existing solutions (e.g.,[1]-[9],[10]-[18]) are mostly limited to a single-user setting, where a single user is allowed to perform encrypted keyword queries on encrypted data. In cloud storage, data owners upload their data to commercial cloud servers and wish to share the data with multiple users, rather than a single user. In this case, searchable encryption works in a multi-user setting.

Schemes used in a single-user setting cannot be used in a multi-user setting directly and effectively because of the increasing requirements of the latter. For example, user dynamics is not considered in a single-user setting but it is important in a multi-user setting. We consider a situation where a data owner stores his data in cloud servers and authorizes a group of subscribed users to access the data for a particular period of time. If

* Corresponding Author 
any of these subscribed users becomes compromised or is no longer qualified to access the data, the data owner would like to revoke their access to the data. If a scheme in a single-user setting is directly used in this case, a key renewal is required among non-revoked users, and re-encryption of the outsourced data is needed. This requirement causes a great deal of performance overhead and is practically almost impossible.

In this paper, we propose a searchable encryption scheme called SEMU in a multi-user setting for cloud storage. We consider an application scenario where an encrypted data sharing system is stored in cloud servers for a number of authorized users. Our contributions are as follows:

1)We first define the SEMU and provide an efficient construction by combining Chinese Remainder Theorem with Public Encryption with Keyword Search(PEKS) [7]. We then prove that the SEMU is secure under Bilinear Diffie-Hellman and Hash Diffie-Hellman assumptions. In the SEMU, data owners, who generate encrypted data and outsource the encrypted data to cloud servers, authorize a group of users to share the data. The authorized users can perform encrypted keyword queries on the encrypted data as well as access the data, but cloud servers have no idea of any information about both the specified keywords and the data. Therefore, the SEMU realizes data privacy and privacy-preserving queries in a multi-user setting for cloud storage.

2)The SEMU supports not only multi-user searchable encryption, but also dynamic changes in authorized users. More importantly, the SEMU does not rely on a shared key to realize multi-user searchable encryption and user dynamics. Each authorized user has a unique key and can perform encrypted keyword queries on encrypted data as well as access data without knowledge of the keys of other users, such that when some users are added or removed, other users are unaffected. In addition, the SEMU performs scalable encryption on cloud server side without shipping the outsourced data back to data owner when some users are added or removed, thus reducing communication overhead.

3)The SEMU can provide privacy-preserving queries, controlling queries, unforgeable queries, and a designated tester, which means that the SEMU possesses features similar to that of most existing schemes in a single-user setting(e.g., [9, 10]) and does not downgrade because of supporting multiple users. Moreover, the SEMU outperforms some schemes in a multi-user setting (e.g., $[6,20,21]$ ) in terms of the ciphertext size of the encrypted keyword and the storage overhead for both the cloud server and the user.

The remainder of this paper is organized as follows: Section 2 presents related work, and Section 3 describes some definitions and other useful concepts that are used in our approach. We construct the SEMU in Section 4 and give the security proof and the performance analysis in Section 5. Finally, the concluding remarks are given in Section 6.

\section{Related Work}

Numerous works have been conducted on searchable encrypted data. Song et al. [1] first proposed a practical scheme for searching on encrypted data. Their scheme considers that a user uploads private data to an untrusted server and keeps the data private from the server administrator, and the user can retrieve the data containing a particular keyword from the server. Subsequently, several works $[2,3,4,5]$ were introduced to improve the efficiency and security of the system. However, the previous works only support a single keyword search in symmetric key setting and cannot be used for some practical applications, such as an email routing system [6]. In 2005, Boneh et al. [7] first proposed PEKS. Their scheme provides a mechanism that enables a message sender to store an encrypted message with some encrypted keywords at a server, where encryption is based on the public key of a receiver. The receiver may then send a trapdoor corresponding to the keywords and to his private key to the server, such that the server can test whether the encryption data and the trapdoor are made with the same key-words, but the server does not learn anything about both the query and the data. The PEKS uses asymmetric instead 
of symmetric encryption and can be used for an email routing system. However, the PEKS has utilized a secure channel between the receiver and the server, which is certainly not suitable for some applications as building a secure channel is usually expensive [8]. Then Baek et al. [8] proposed a searchable public-key encryption for a designated tester (dPEKS) to remove the secure-channel assumption of the PEKS. In the dPEKS, only the server can test whether or not a given dPEKS ciphertext is related with a trapdoor by using its private key. Later, several variants (e.g., [9]-[15]) of the PEKS have been proposed with various improvements. Recently, Liu et al. [16, 17] proposed privacy preserving keyword search schemes and applied them to cloud storage. Their schemes support searchable encrypted files, and the server participates in partial decipherment to reduce a client's computational overhead. Cheng et al. [18] also proposed a privacy preserving keyword search for cloud storage. However, the aforementioned schemes support only a designated receiver, and do not support multiple designated receivers. In other words, these schemes are constructed in a single-user setting.

To support multi-user searches on encrypted data, Curtmola et al. [19] transferred their searchable encryption scheme for a single-user setting to one for a multi-user setting. However, their scheme shares the same secret key among all users to search over encrypted database. This approach is evidently unscalable for large and dynamic systems where user addition and revocation may occur frequently. Moreover, user revocation in their scheme is based on broadcast encryption, where a revocation affects all non-revoked users. Hwang and Lee [6] proposed searchable public key encryption in a multi-user setting. Their scheme enables multiple users to search over encrypted data and does not share the same secret key among all users. However, in their scheme, the ciphertext size is proportional to the number of receivers. Moreover, user revocation is not considered. Bao et al. [20, 21] proposed multi-user private keyword search schemes for cloud computing. Their schemes enable each user to possess a distinct query key to construct search queries, but all authorized users share the same key to generate the index for a keyword $w$. This requirement results in a weakness that a revoked user can write records to the dataset. In addition, their schemes need to store a U-Hkey (containing user identities and helper keys). To revoke a user $u$, the data owner instructs the cloud server to delete the help key $h k_{u}$ of the user $u$ from the U-Hkey list maintained by the cloud server. In other words, user revocation mainly depends on whether the cloud server removes the user help key $h k_{u}$ from the U-Hkey list. However, the cloud service provider is not to be fully-trusted and may not follow the instruction of the data owner to remove the help key $h k_{u}$ from the U-Hkeylist to make a benefit. For example, to continue to access the data, a revoked user may give some benefits to the cloud service provider, such that the cloud service provider may not remove his help key from the U-Hkeylist. This practice negatively affects the data owner, but the data owner cannot prevent this situation from occurring. In addition, all authorized users in their schemes share the same decrypted key.

\section{Preliminaries and Definition}

In this section we describe some definitions and other useful concepts that are used in our approach.

\subsection{Preliminaries}

Bilinear Pairings. Let $G$ and $G_{T}$ be two multiplicative cyclic groups of prime order $p, g$ be a generator of $G$. The bilinear map $\hat{e}: G \times G \rightarrow G_{T}$ should be satisfied the following properties:

1) Bilinearity: $\hat{e}\left(u^{a}, v^{b}\right)=\hat{e}(u, v)^{a b}$ for $u, v \in G$. 
2) Non-degeneracy: $\hat{e}(g, g) \neq 1$.

3) Computability: There is an efficient algorithm for computing the map $\hat{e}$.

Decisional Diffie-Hellman (DDH) problem)[22]. Decisional Diffie-Hellan (DDH) problem in $G$ is as follows: given $\left(g, g^{a}, g^{b}, g^{c}\right)$ as input, output yes if $c=a b$ and no otherwise. We say that DDH is intractable if all polynomial time algorithms have a negligible advantage in solving DH problem.

Bilinear Diffie-Hellman(BDH) Problem[23]. Given $\left(g, g^{a}, g^{b}, g^{c}\right) \in G$ as input, com-pute $\hat{e}(g, g)^{a b c} \in G_{T}$. We say that BDH problem is intractable if all polynomial time algorithms have a negligible advantage in solving $\mathrm{BDH}$ problem.

Chinese remainder theorem[24]. Let $p_{1}, p_{2}, \ldots, p_{n}$ be positive integers that are relatively prime in pairs. Then, for any given integers $b_{1}, b_{2}, \ldots, b_{n}$, the system of congruences

$$
y \equiv b_{i} \bmod p_{i}(1 \leq i \leq n)
$$

has a unique solution modulo $N=p_{1} p_{2} \ldots p_{n}$. The solution is given by

$$
y=\sum_{i=1}^{n} b_{i} N_{i} N_{i}^{\prime} \bmod N
$$

where $N_{i}=N / p_{i}$ and $N_{i} N_{i}^{\prime} \equiv 1 \bmod p_{i}$.

\subsection{System Model}

Three types of entities are used in the proposed system: data owner, cloud server, and multiple users. The data owner outsources data in an encrypted form to the cloud server and authorizes a group of users to access the data. The SEMU is a mechanism that enables only authorized users to generate valid encrypted keyword queries, and only the cloud server can verify whether encrypted data contain the encrypted keywords while learning nothing else. In this work, we assume that the cloud service provider is honest but curious, and should be trusted to perform searchable encryption and re-encryption operations correctly when the cloud server provider is not profitable.

Let $m$ be the number of authorized users. The SEMU is consisted of nine polynomial-time algorithms as follows.

1) Global $\operatorname{Set} \operatorname{up}(\lambda)$ : Takes a security parameter $\lambda$ as input, this algorithm generates a global parameter $g p$.

2) KeyGen(gp): Takes the global parameter $g p$ as input, this algorithm outputs the private/public key pairs $\left(s k_{s}, p k_{s}\right)$ of the cloud server, and $\left(s k_{d}, p k_{d}\right)$ of the data owner as well as $\left(s k_{i}, p k_{i}\right)$ of authorized user $u_{i}$, where $i=1,2, \ldots, m$.

3) EnKeyword $\left(g p, p k_{1}, \ldots, p k_{m}, w\right)$ : This algorithm is executed by the data owner to encrypt a keyword $w$. Takes as input the global parameter $g p$, the public keys $\left(p k_{1}, \ldots, p k_{n}\right)$ of the authorized users and a keyword $w$, it outputs a searchable keyword ciphertext $C_{1}=[A, B]$ with the public keys $\left(p k_{1}, \ldots, p k_{n}\right)$.

4) EnData( $\left.g p, p k_{1}, \ldots, p k_{n}, M\right)$ : This algorithm is executed by the data owner to encrypt data $M$. Takes as input the global parameter $g p$, the public keys $\left(p k_{1}, \ldots, p k_{m}\right)$ of the authorized users and data $\mathrm{M}$, this algorithm outputs a ciphertext $C_{2}=[D, E]$ with the public keys $\left(p k_{1}, \ldots, p k_{n}\right)$.

5) Trapdoor $\left(p p, s k_{i}, p k_{s}, w\right)$ : This algorithm is executed by an authorized user to generate a trapdoor. Takes as input the global parameter $g p$, the private key $s k_{i}$ of the 
authorized user, the public key $p k_{s}$ of the cloud server and a keyword $w$, it outputs a trapdoor $T_{w}$ for the keyword search over encrypted data.

6) Test $\left(g p, C_{1}, T_{w}\right)$ : This algorithm is executed by the cloud server to search the encrypted data with a keyword $w$. It takes as input the global parameter $g p$, a searchable keyword ciphertext $C_{1}$ and a trapdoor $T_{w}$, it outputs '1' if the ciphertext $C_{1}$ includes the keyword $w$, otherwise, this algorithm outputs ' 0 '.

7) DeData $\left(g p, A, C_{2}, s k_{i}\right)$ : This algorithm is executed by a authorized user. Takes as input the global parameter $g p$, the ciphertexts $\left(A, C_{2}\right)$ and the private key $s k_{i}$ of the authorized user. This algorithm outputs the data $M$.

8) Addition $\left(g p, u_{l}\right)$ : This algorithm is executed by the data owner to add a new authorized user $u_{l}$ to the system.

9) Revocation $\left.g p, u_{y}, s k_{d}, s k_{s}, p k_{1}, \ldots, p k_{m}\right)$ : This algorithm is executed together by both the data owner and the cloud server to revoke a authorized user $u_{\gamma}$ from the system. Takes as input the global parameter $g p$, the private key $s k_{d}$ of the data owner, the private key $s k_{s}$ of the cloud server and the public keys $\left(p k_{1}, \ldots, p k_{n}\right)$ of the authorized users. This algorithm revokes the authorized user $u_{\gamma}$ from the system.

\subsection{Security Definition}

In this section, we define the security for the SEMU in the sense of semantic security. According to the definition of the SEMU, it consists of two encryption algorithms, i.e., EnKeyword() and EnData(.). Therefore, we first define the security for the EnKeyword() and the EnData(.), and then give the security definition of the SEMU.

Semantic Security of EnKeyword(.): The algorithm EnKeyword() is semantically secure against an adaptive chosen keyword attack if for any polynomial-time adversary, $A_{1}$, the advantage $\operatorname{Adv}_{A_{1}}^{I N D-C P A}(\lambda)$ is negligible in following Game 1:

1) Setup: The adversary $A_{1}$ generates his private/public key pairs $\left(s k_{A_{1}}, p k_{A_{1}}\right)$, and gives $p k_{A_{1}}$ to an algorithm $B_{1}$. The algorithm $B_{1}$ generates the private/public key pairs $\left(s k_{1}, p k_{1}\right), \ldots,\left(s k_{m}, p k_{m}\right)$ of authorized users and then gives $\left(p k_{1}, \ldots, p k_{m}\right)$ to the adversary $A_{1}$.

2) Phase 1 (Trapdoor queries): The adversary $A_{1}$ can adaptively asks $B_{1}$ for the trapdoor $T_{w}$ for any keyword $w \in\{0,1\}^{*}$ of his choice.

3) Challenge: The adversary $A_{1}$ gives $B_{1}$ two keywords, $w_{0}$ and $w_{1}$ on which it wishes to be challenged. The restriction is that $A_{1}$ did not previously ask for the trapdoors $T_{w_{0}}$ and $T_{w_{1}}$. $B_{1}$ picks a random $\beta_{1} \in\{0,1\}$ and computes a ciphertext $C_{1}^{*}=\left[A^{*}, B^{*}\right]$, and sends $C_{1}^{*}$ to $A_{1}$.

4) Phase 2 (Trapdoor queries): The adversary $A_{1}$ can adaptively asks $B_{1}$ for the trapdoor $T_{w}$ for any keyword $w \in\{0,1\}^{*}$ of his choice as long as $w \neq w_{0}, w_{1}$.

5) Guess: The adversary $A_{1}$ outputs $\beta_{1}^{\prime} \in\{0,1\}$ and wins the Game 1 if $\beta_{1}=\beta_{1}^{\prime}$.

We define the adversary $A_{1}$ 's advantage in breaking the EnKeyword() as

$$
\left.A d_{A_{1}^{N}}^{L N D C P}(\lambda)=\mid \mathrm{P} \mathrm{r} \beta_{1}=\beta_{1}^{\prime}\right]-\frac{1}{2} \mid
$$


Semantic Security of $\operatorname{EnData(.):~The~algorithm~EnData(.)~is~semantically~secure~}$ against an adaptive chosen plaintext attack if for any polynomial time adversary, $A_{2}$, the advantage $A d v_{A_{2}}^{I N-C P A}(\lambda)$ is negligible in following Game 2:

1) Setup: Algorithm $B_{2}$ is given the public key $p k_{A_{2}}$ of the adversary $A_{2}$. The algorithm $B_{2}$ generates the private/public key pairs $\left(s k_{1}, p k_{1}\right), \ldots,\left(s k_{m}, p k_{m}\right)$ of authorized users and then gives $\left(p k_{1}, \ldots, p k_{n}\right)$ to the adversary $A_{2}$.

2) Challenge: The adversary $A_{2}$ gives $B_{2}$ two plaintexts, $M_{0}$ and $M_{1}$ on which it wishes to be challenged. The restriction is that $A_{2}$ did not previously ask for the plaintexts $M_{0}$ and $M_{1}$. $B_{2}$ picks a bit $\beta_{2} \in\{0,1\}$ and computes $\left(A^{*}, C_{2}^{*}=\left[D^{*}, E^{*}\right]\right)$, and sends the ciphertexts $\left(A^{*}, C_{2}^{*}\right)$ to $A_{2}$.

3) Guess: The adversary $A_{2}$ outputs $\beta_{2}^{\prime} \in\{0,1\}$ and wins the Game 2 if $\beta_{2}=\beta_{2}^{\prime}$.

We define the adversary $A_{2}$ 's advantage in breaking the EnData(.) as

$$
\left.A d_{A_{2}^{N}}^{I N E C P}(\lambda)=\mid \mathrm{P} \mathrm{r} \beta_{2}=\beta_{2}^{\prime}\right]-\frac{1}{2} \mid
$$

Semantic Security of the SEMU: Given the polynomial time adversary $A$ consisting of the adversary $A_{1}$ and the adversary $A_{2}$, where the adversary $A_{1}$ initiates attacks on the EnKeyword() and the adversary $A_{2}$ initiates attacks on the EnData(.). We say that the SEMU scheme is semantically secure if the adversary $A$ 's advantage

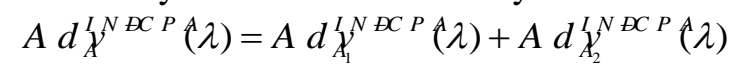

is negligible.

\section{Construction}

In this section, we present a concrete construction of the SEMU. The construction is based on both the Chinese Remainder Theorem and the PEKS [7].

Let $U=\left(u_{1}, u_{2}, \ldots, u_{m}\right)$ be a set of authorized users, the SEMU includes following several sections.

1) Global $\operatorname{Setup}(\lambda)$ : Choose a set of positive integers $\left(p_{1}, p_{2}, \ldots, p_{n}\right)$ that are relatively prime in pairs and $p_{1}<p_{2}<\ldots<p_{n}(n>m>2)$. Let $G$ and $G_{T}$ be two multiplicative cyclic groups of prime order $p$, and $g \in G$ be a generator. Where $p$ is larger enough to guarantee that the discrete logarithm problem in $G$ is intractable and $p>p_{n}$. Further let $\hat{e}$ be the bilinear map $\hat{e}: G \times G \rightarrow G_{T}$. Also assuming $H: G \rightarrow Z_{p_{1}}^{*}$, $H_{1}:\{0,1\}^{*} \rightarrow G$, and $H_{2}: G_{T} \rightarrow Z_{p_{1}}^{*}$ be hash functions that are modeled as a random.

Given a security parameter $\lambda$, this algorithm returns a global parameter $g p=\left(g, G, G_{T}, \hat{e}, p, p_{1}, \ldots, p_{m}, H_{1}(),. H_{2}(),. H().\right)$.

2) KeyGen(gp): Takes as input $g p$, this algorithm chooses randomly integers $\left(\zeta, \tau, k_{i}\right) \in Z_{p}^{*}$, and computes $p k_{s}=g^{\zeta} \bmod p, p k_{d}=g^{\tau} \bmod p, p k_{i}=g^{k_{i}} \bmod p$, where $i=1,2, \ldots, m$. Finally this algorithm outputs $s k_{d}=\tau$ to the data owner secretly, $s k_{s}=\zeta$ to the cloud server secretly, and $s k_{i}=k_{i}$ to authorized user $u_{i}$ secretly. This algorithm publishes $p k_{\mathrm{s}}, p k_{\mathrm{d}}, p k_{1}=\left(p k_{1}, p_{1}\right), \ldots, p k_{\mathrm{m}}=\left(p k_{m}, p_{\mathrm{m}}\right)$ as public information.

3) EnKeyword $\left(g p, p k_{1}, \ldots, p k_{n}, w\right)$ : This algorithm is executed by the data owner to encrypt a keyword $w$. Takes as input $g p$, the public keys $\left(p k_{1}, \ldots, p k_{m}\right)$ of the 
authorized users and a keyword $w$. This algorithm chooses a random integer $r \in Z_{p}^{*}$, and computes

$$
\begin{gathered}
N=\prod_{i=1}^{m} p_{i}=\prod_{i \in U} p_{i}, \quad N_{i}=N / p_{i}, \quad N_{i} N_{i}^{\prime}=1 \bmod p_{i}, \\
B_{i}=H_{2}\left(\hat{e}\left(p k_{i}^{r}, H_{1}(w)\right)\right), \\
A=g^{r} \operatorname{mog} \phi, \quad B=\sum_{i \in U} B_{i} N_{i} N_{i}^{\prime} \bmod N .
\end{gathered}
$$

This algorithm outputs $C_{1}=[A, B]$ to the cloud server.

4) EnData $\left(g p, p k_{1}, \ldots, p k_{n}, M\right):$ This algorithm is executed by the data owner to encrypt data $M$. Takes as input $g p$, the public keys $\left(p k_{1}, \ldots, p k_{n}\right)$ of the authorized users and data $M$, this algorithm chooses a random integer $x \in Z_{p_{1}}^{*}$, and encrypts data $M$ as following

$$
\begin{gathered}
N=\prod_{i=1}^{m} p_{i}=\prod_{i \in U} p_{i}, N_{i}=N / p_{i}, \quad N_{i} N_{i}^{\prime}=1 \bmod p_{i}, \\
\sigma_{i}=H\left(p k_{i}^{r}\right), D=x \sum_{i \in U} \sigma_{i} N_{i} N_{i}^{\prime} \bmod N, E=M \oplus H(x) .
\end{gathered}
$$

This algorithm outputs $C_{2}=[D, E]$ to the cloud server. Where $r$ is the value selected in the EnKeyword() phase.

5) Trapdoor $\left(g p, s k_{i}, p k_{s}, w\right)$ : This algorithm is executed by an authorized user to generate a trapdoor for the keyword $w$. Takes as input $g p$, the private key $s k_{i}$ of an authorized user, the public key $p k_{s}$ of the cloud server and a keyword $w$. This algorithm chooses a random integer $\alpha \in Z_{p}^{*}$, and computes

$$
T_{1}=g^{\alpha} \mathrm{mog}, T_{2}=H_{1}(w)^{s k_{i}} p k_{s}^{\alpha}, T_{3}=p_{i}
$$

This algorithm outputs a trapdoor $T_{w}=\left[T_{1}, T_{2}, T_{3}\right]$ to the cloud server.

6) Test $\left(g p, C_{1}, T_{w}\right)$ : This algorithm is executed by the cloud server to search encrypted data with a keyword. It takes as input $g p$, the private key $s k_{s}$ of the cloud server, the searchable keyword ciphertext $C_{1}$ and the trapdoor $T_{w}$. This algorithm computes

$$
\begin{gathered}
T=T_{2} / T_{1}^{s k_{s}}=H_{1}(w)^{s k_{i}}, \\
B_{i}=B \bmod p_{i}=H_{2}\left(\hat{e}\left(p k_{i}^{r}, H_{1}(w)\right)\right),
\end{gathered}
$$

and checks if $B_{i}=H_{2}(\hat{e}(T, A))$. If the equality is satisfied, then this algorithm outputs '1'; otherwise, outputs ' 0 '. If the algorithm outputs ' 1 ', then the cloud server sends $\left(A, C_{2}\right)$ to the query user.

\section{Correctness:}

When assuming $\left(B_{i}=H_{2}\left(\hat{e}\left(p k_{i}^{r}, H_{1}(w)\right)\right), A=g^{r}\right)$ are valid for the keyword $w$ and the trapdoor $\left(T_{1}=g^{\alpha} \bmod p, T_{2}=H_{1}(w)^{s_{i}} p k_{s}^{\alpha}\right)$, the correctness of the Test(.) algorithm is verified as

$$
T=T_{2} / T_{1}^{s k_{s}}=H_{1}(w)^{s k_{i}}, H_{2}(\hat{e}(T, A))=H_{2}\left(\hat{e}\left(H_{1}(w)^{s k_{i}}, g^{r}\right)=B_{i} .\right.
$$

7) DeData $\left(g p, A, C_{2}, s k_{i}\right)$ : This algorithm is executed by an authorized user. Takes as input $g p$, the ciphertexts $\left(A, C_{2}\right)$ and the private key $s k_{i}$ of the authorized user. This algorithm computes

$$
x=D H\left(A^{s k_{i}}\right)^{-1} \bmod p_{i}, \quad M=E \oplus H(x) .
$$




\section{Correctness:}

When assuming $\left(D=x \sum_{i \in U} \sigma_{i} N_{i} N_{i}^{\prime} \bmod N, A=g^{r}, E=M \oplus H(x)\right)$ are valid for the data $M$, the correctness of the DeData(.) algorithm is verified as

$$
\sigma_{i}=H\left(p k_{i}^{r}\right), \quad D H\left(A^{s k_{i}}\right)^{-1} \bmod p_{i}=x H\left(p k_{i}^{r}\right) H\left(p k_{i}^{r}\right)^{-1}=x
$$

8) Addition $\left(g p, u_{l}\right)$ : This algorithm is executed by the data owner to add a new authorized user $u_{l}$ to the system. Taking as input $g p$, this algorithm runs the KeyGen(.) algorithm to generate the public key $p k_{l}=\left(p k_{l}, p_{l}\right)$ of the new authorized user. This algorithm computes

$$
\begin{gathered}
B_{l}=H_{2}\left(\hat{e}\left(p k_{l}^{r}, H_{1}(w)\right)\right), \\
\bar{N}=\prod_{i \in\left(U \cup u_{l}\right)} p_{i}, \quad \bar{N}_{i}=\bar{N} / p_{i}, \bar{N}_{i} \bar{N}_{i}^{\prime}=1 \bmod p_{i}, \\
\bar{B}=\sum_{i \in\left(U \cup u_{l}\right)} B_{i} \bar{N}_{i} \bar{N}_{i}^{\prime} \operatorname{mos} \overline{\mathbb{N}},
\end{gathered}
$$

and then outputs $\bar{C}_{1}=[A, \bar{B}]$ to the cloud server.

9) Revocatior $\left.g p, u_{y}, s k_{d}, s k_{s}, p k_{1}, \ldots, p k_{m}\right)$ : This algorithm is executed by both the data owner and the cloud server to revoke an authorized user $u_{\gamma}$ from the system. The data owner chooses randomly integers $\tilde{r} \in Z_{p}^{*}$ and $\tilde{x} \in Z_{p_{1}}^{*}$, then computes

$$
\begin{gathered}
\tilde{N}=\prod_{i \in\left(U \backslash u_{\gamma}\right)} p_{i}, \tilde{N}_{i}=\tilde{N} / p_{i}, \tilde{N}_{i} \tilde{N}_{i}^{\prime}=1 \bmod p_{i}, \\
\tilde{\sigma}_{i}=H\left(p k_{i}^{\tilde{r}}\right), \quad \tilde{D}=\tilde{x} \sum_{i \in\left(U \backslash u_{\gamma}\right)} \tilde{\sigma}_{i} \tilde{N}_{i} \tilde{N}_{i}^{\prime} \bmod \tilde{N}, \\
\tilde{B}_{i}=H_{2}\left(\hat{e}\left(p k_{i}^{\tilde{r}}, H_{1}(w)\right)\right), \quad \tilde{B}=\sum_{i \in\left(U \backslash u_{\gamma}\right)} \tilde{B}_{i} \tilde{N}_{i} \tilde{N}_{i}^{\prime} \bmod \tilde{N}, \\
\tilde{A}=g^{\tilde{r}} \mathrm{mo} \phi, R=H(x) \oplus H(\tilde{x}), \quad \mu=p k_{s}^{s k_{d}} \bmod p, \quad v=E_{\mu}(R) .
\end{gathered}
$$

then sends $\left(\tilde{C}_{1}=[\tilde{A}, \widetilde{B}], \tilde{C}_{2}=[\tilde{D}, \tilde{E}], v\right)$ to the cloud server. Where $E_{\mu}($.$) and D_{\mu}($. denote a symmetrical encryption and decryption operations with a key $\mu$ such as Advanced Encryption Standard(AES) algorithm.

Receiving $\left(\tilde{C}_{1}=[\tilde{A}, \tilde{B}], \widetilde{C}_{2}=[\tilde{D}, \tilde{E}], v\right)$, the cloud server computes

$$
\mu=p k_{d}^{\text {sks }} \bmod p, R=D_{\mu}(v), \tilde{F}=F \oplus R=M \oplus H(\tilde{x}) .
$$

and replaces the old $\left(C_{1}, C_{2}\right)$ with the new $\left(\tilde{C}_{1}, \tilde{C}_{2}\right)$.

\section{Security and Performance}

\subsection{Security}

We now prove the security of the SEMU scheme under the BDH and the DDH assumption described above in the same way as [7].

Theorem 1: EnKeyword() is semantically secure against a chosen keyword attack in the Game 1 under random oracle model assuming the BDH is intractable.

Proof: Assume that $A_{1}$ is an adversary with advantage $\varepsilon_{1}$ in breaking the EnKeyword() against IND-CPA, and $H_{1}$ and $H_{2}$ are modeled as random oracles. Suppose $A_{1}$ makes at most $q_{H_{2}}>0$ hash function queries to $H_{2}$ and at most $q_{T}>0$ trapdoor queries. Then there is an algorithm $B_{1}$ that solves the BDH problem with 
probability at least $\varepsilon_{1}^{\prime}=\varepsilon_{1} / e q_{T} q_{H_{2}}$ and a running time $O\left(\operatorname{time}\left(A_{1}\right)\right)$, where $e$ is the base of the natural logarithm. Notice that if the BDH assumption holds in $G$ then $\varepsilon_{1}^{\prime}$ is a negligible function and consequently $\varepsilon_{1}$ must be a negligible function in the security parameter.

The algorithm $B_{1}$ is given $u_{1}=g^{a} \in G, u_{2}=g^{b} \in G, u_{3}=g^{c} \in G$. Its goal is to output $v=\hat{e}(g, g)^{a b c} \in G_{T}$. The algorithm $B_{1}$ simulates a challenger and interacts with the adversary $A_{1}$.

1) Setup: The adversary $A_{1}$ generates his public key such that $p k_{A_{1}}=u_{1}=g^{a}$ and gives $p k_{A_{1}}$ to the algorithm $B_{1}$. To simulate the public/private key pairs $\left(p k_{1}, s k_{1}\right), \ldots,\left(p k_{m}, s k_{m}\right)$ of authorized users, the algorithm $B_{1}$ randomly chooses $\left(\delta_{1}, \ldots, \delta_{m}\right) \in Z_{p}^{*}$, and then lets $p k_{1}=\left(u_{1}^{\delta_{1}}, p_{1}\right), \ldots, p k_{\mathrm{m}}=\left(u_{\mathrm{m}}^{\delta_{m}}, p_{\mathrm{m}}\right)$. Finally, the $B_{1}$ gives $\left(p k_{1}, \ldots, p k_{n}\right)$ to the adversary $A_{1}$.

$H_{1}$-Queries: To respond to $H_{1}$ queries, the algorithm $B_{1}$ maintains a $H_{1}$-list $=<w_{i}, h_{i}, a_{i}, c_{i}>$, which is initially empty. When the adversary $A_{1}$ queries $H_{1}$ at a point $w \in\{0,1\}^{*}, B_{1}$ checks if the query $w_{i}$ already appears in the $H_{1}$-list. If it appears, $B_{1}$ responds with $H_{1}\left(w_{i}\right)=h_{i} \in G$. Otherwise, $B_{1}$ generates a random coin $c_{i} \in\{0,1\}$, so that $\operatorname{Pr}\left[c_{i}=0\right]=1 /\left(q_{T}+1\right)$. If $c_{i}=0, B_{1}$ computes $h_{i}=u_{2}^{a_{i}}=g^{b a_{i}}$, where $a_{i} \in Z_{p}^{*}$ is a random value. If $c_{i}=1, B_{1}$ computes $h_{i}=g^{a_{i}} . B_{1}$ adds $<w_{i}, h_{i}, a_{i}, c_{i}>$ to the $H_{1}$-list and responds to $A_{1}$ by setting $H_{1}\left(w_{i}\right)=h_{i}$.

$H_{2}$-Queries: To respond to $H_{2}$ queries, $B_{1}$ maintains a $H_{2}$-list $=<t_{i}, v_{i}>$, which is initially empty. When $A_{1}$ issues a query $t_{j} \in G_{T}$ to $H_{2}, B_{1}$ checks if $t_{j}$ is already on the $H_{2}$-list. If so, $B_{1}$ responds to $A_{1}$ with $H_{2}\left(t_{j}\right)=v_{j}$. Otherwise, $B_{1}$ responds to a query for $H_{2}\left(t_{j}\right)$ by picking a random value $v_{j} \in Z_{p_{1}}^{*}$ for the $t_{j}$ and setting $H_{2}\left(t_{j}\right)=v_{j}$, and adds $\left\langle t_{j}, v_{j}>\right.$ to the $H_{2}$-list.

2) Trapdoor queries. When the adversary $A_{1}$ issues a query for the trapdoor corresponding to a keyword $w_{i}, B_{1}$ obtains $\left\langle w_{i}, h_{i}, a_{i}, c_{i}\right\rangle$ from the $H_{1}$-list. If $c_{i}=0, B_{1}$ reports failure and terminates. Otherwise, $B_{1}$ computes $T_{w}=u_{1}^{a_{i} \delta_{i}}$ and responds $T_{w}$ to $A_{1}$. By the setting of $p k_{i}=g^{a \delta_{i}}$ in the Setup phase, and $H_{1}\left(w_{i}\right)^{s k_{i}}=g^{a a_{i} \delta_{i}}$ is satisfied, therefore $T_{w}$ is the correct trapdoor for the keyword $\mathrm{w}$ under the public key $p k_{i}=g^{a \delta_{i}}$.

3) Challenge: The adversary $A_{1}$ gives $B_{1}$ two keywords $w_{0}$ and $w_{1}$, which $A_{1}$ wishes to be challenged on. The restriction is that $A_{1}$ did not previously ask for the trapdoors corresponding to the keyword $w_{0}$ and $w_{1}$. $B_{1}$ obtains $<w_{0}, h_{0}, a_{0}, c_{0}>$ and $\left.<w_{1}, h_{1}, a_{1}, c_{1}\right\rangle$ from the $H_{1}$-list. If $c_{0}=1$ and $c_{1}=1, B_{1}$ reports failure and terminates. Otherwise, $B_{1}$ chooses $\beta_{1} \in\{0,1\}$ such that $c_{\beta_{1}}=0$ and responds $C_{1}^{*}=\left[A^{*}, B^{*}\right]$ such that $A^{*}=u_{3}=g^{c} \bmod p, B_{i}^{*}=z_{i}, B^{*}=\sum_{i \in U} B_{i}^{*} N_{i} N_{i}^{\prime} \bmod N$, where $N=\prod_{i \in U} p_{i}, N_{i}=N / p_{i}, \quad N_{i} N_{i}^{\prime}=1 \bmod p_{i}, \quad z_{i} \in Z_{p_{1}}^{*}$ is a random value. Notice that $B_{i}^{*}=B \bmod p_{i}=z_{i}$ and $H_{2}\left(\hat{e}\left(T_{w_{\beta_{1}}}, A\right)\right)=H_{2}\left(\hat{e}\left(\left(g^{b a_{i}}\right)^{s k_{i}}, u_{3}\right)\right)=H_{2}\left(\hat{e}(g, g)^{a b c a_{i} \delta_{i}}\right)$. 
4) Guess: The adversary $A_{1}$ outputs $\beta_{1}^{\prime}, B_{1}$ picks a $<t_{i}, v_{i}>$ from the $H_{2}$-list, gives $t_{i}^{1 / a_{i} \delta_{i}}$ as its guess for $\hat{e}(g, g)^{a b c}$.

To show that $B_{1}$ correctly outputs $\hat{e}(g, g)^{a b c}$ with a probability of at least $\varepsilon_{1}^{\prime}$, we analyze the probability that $B_{1}$ does not abort during the simulation.

We assume that the adversary $A_{1}$ does not ask for the trapdoor of the same keyword twice. Since no matter whether $c_{i}=0$ or $c_{i}=1$, the distribution on $H_{1}\left(w_{i}\right)$ is the same, and the value of $c_{i}$ is independent of $A_{1}$ 's view, therefore, one trapdoor causes $B_{1}$ to report "failure" is with probability $1 /\left(q_{T}+1\right)$. Since $A_{1}$ makes at most $q_{T}$ trapdoor queries, the probability that $B_{1}$ does not abort in all trapdoor queries is $\left(1-1 /\left(q_{T}+1\right)^{q_{T}}\right) \geqslant 1 / e$.

The algorithm $B_{1}$ will abort during the challenge phase if $A_{1}$ issues the trapdoor queries about $w_{0}$ and $w_{1}$ with $c_{0}=c_{1}=1$. Since $A_{1}$ has not queried for the trapdoor for $w_{0}$ and $w_{1}$, both $c_{0}$ and $c_{1}$ are independent of $A_{1}$ 's current view. In addition, the two values are independent of one another. Therefore, the probability that $B_{1}$ does not abort in Challenge is at least $1 / q_{T}$. Since $A_{1}$ can never issue a trapdoor query for the keywords $w_{0}$ and $w_{1}$, the probability that $B_{1}$ does not abort is at least $1 / e q_{T}$.

Following we show that $B_{1}$ outputs the solution to the given the BDH instance with probability at least $\varepsilon_{1} / q_{\mathrm{H}_{2}}$.

Let $\varepsilon$ be the event that $A_{1}$ does not issue a query for either one of $H_{2}\left(\hat{e}\left(p k_{i}^{r}, H_{1}\left(w_{0}\right)\right)\right)$ or $H_{2}\left(\hat{e}\left(p k_{i}^{r}, H_{1}\left(w_{1}\right)\right)\right)$. Because $C_{1}^{*}$ is an encryption of $w_{0}$ or $w_{1}$ is independent of $A_{1}$ 's view, $A_{1}$ 's output $\beta_{1}^{\prime}$ will satisfy $\beta_{1}=\beta_{1}^{\prime}$ with probability at most $1 / 2$. By definition of the security game, we know that $\left|\operatorname{Pr}\left[\beta_{1}=\beta_{1}^{\prime}\right]-(1 / 2)\right| \geq \varepsilon_{1}$, and $\left.\operatorname{Pr}\left[\beta_{1}=\beta_{1}^{\prime}\right] \leq(1 / 2)+(1 / 2) \operatorname{Pr}[\urcorner \varepsilon\right], \quad \operatorname{Pr}\left[\beta_{1}=\beta_{1}^{\prime}\right] \geq(1 / 2)-(1 / 2) \operatorname{Pr}[\ulcorner\varepsilon]$. Therefore, $\operatorname{Pr}\left[\ulcorner\varepsilon] \geq 2 \varepsilon_{1}\right.$. Since $B_{1}$ will choose the correct pair in $H_{2}$-list with the probability at least $1 / q_{H_{2}}$, then produces the correct answer with the probability at least $\varepsilon_{1} / q_{H_{2}}$.

Because $B_{1}$ does not abort with probability at least $1 / e q_{T}, B_{1}$ 's success probability overall is at least $\varepsilon_{1} / e q_{T} q_{H_{2}}$ as required.

Theorem 2: $\mathrm{En} \mathrm{D}$ a $\mathrm{t}$ : is semantically secure against a chosen plaintext attack in the Game 2 under assuming the Decisional Diffie-Hellman (DDH) is intractable.

Proof: Assume that $A_{2}$ is an adversary with advantage $\varepsilon_{2}$ in breaking the EnData(.) against IND-CPA. We construct a algorithm $B_{2}$ which has an advantage $\varepsilon_{2}^{\prime}=\varepsilon_{2}$ in solving the $\mathrm{DDH}$ problem in $G$.

The algorithm $B_{2}$ is given a random DDH challenge $\left(g, g^{a}, g^{b}, g^{c}\right)$ and $H: G \rightarrow Z_{p_{1}}^{*}$, its goal is to guess $c=a b$ when $\beta_{2}=0$ and $c$ is a random values from $Z_{p}^{*}$ otherwise. Where $\beta_{2}$ is a bit $\in\{0,1\}$, and $(a, b)$ are randomly and independently chosen from $Z_{p}^{*}$.

1) Setup: The algorithm $B_{2}$ is given the adversary $A_{2}$ 's public key $p k_{A_{2}}=g^{a}$. To simulate the public/private key pairs $\left(p k_{1}, s k_{1}\right), \ldots,\left(p k_{m}, s k_{m}\right)$ of authorized users, the algorithm $B_{2}$ randomly chooses $\left(\xi_{1}, \ldots, \xi_{m}\right) \in Z_{p}^{*}$, and lets $p k_{1}=g^{a \xi_{1}}, \ldots, p k_{\mathrm{m}}=g^{a \xi_{m}}$. 
Finally, $B_{2}$ gives $\left(p k_{1}, \ldots, p k_{n}\right)$ to the adversary $A_{2}$. Notice that $p k_{i}=g^{a \xi_{i}}=g^{s k_{i}}$, where $i=1,2, \ldots, m$.

2) Challenge: The adversary $A_{2}$ gives the algorithm $B_{2}$ two plaintexts, $M_{0}$ and $M_{1}$ which it wishes to be challenged on. $B_{2}$ picks a random $\beta_{2} \in\{0,1\}$ and responds with the challenge ciphertext $\left(A^{*}, C_{2}^{*}=\left[D^{*}, E^{*}\right]\right)$ such that

$$
\begin{gathered}
N=\prod_{i=1}^{m} p_{i}=\prod_{i \in U} p_{i}, N_{i}=N / p_{i}, \quad N_{i} N_{i}^{\prime}=1 \bmod p_{i}, \\
A^{*}=g^{b}, D=x \sum_{i \in U} H\left(g^{c \xi_{i}}\right) N_{i} N_{i}^{\prime} \bmod N, E^{*}=M_{\beta_{2}} \oplus H(x)
\end{gathered}
$$

and sends $\left(A^{*}, C_{2}^{*}=\left[D^{*}, E^{*}\right]\right)$ to $A_{2}$. Where $c \in Z_{p}^{*}$ and $x \in Z_{p_{1}}^{*}$ are random values.

3) Guess: The adversary $A_{2}$ outputs $\beta_{2}^{\prime} . B_{2}$ guesses that $\beta_{2}=0$ if and only if $\beta_{2}=\beta_{2}^{\prime}$.

If $\beta_{2}=0$, then $c=a b$ and the ciphertext $\left(A^{*}, C_{2}^{*}\right)$ is a valid ciphertext. Notice that

$$
D^{*} H\left(A^{* s k_{i}}\right)^{-1} \bmod p_{i}=x H\left(g^{c \xi_{i}}\right) H\left(\left(g^{b}\right)^{a \xi_{i}}\right)^{-1}=x H\left(g^{c \xi_{i}}\right) H\left(g^{a b \xi_{i}}\right)^{-1} .
$$

Also when $\beta_{2}=0, B_{2}$ succeeds if and only if $A_{2}$ succeeds, which occurs with probability $\varepsilon_{2}$. Because $\beta_{2}$ is chosen uniformly at random and independent from $A_{2}$ 's view, and $A_{2}$ has no information about the value of $\beta_{2}$ from $\left(A^{*}, C_{2}^{*}\right)$, so the probability that $B_{2}$ succeeds ( $A_{2}$ succeeds when $\beta_{2}=\beta_{2}^{\prime}$ ) is

$$
\left.\operatorname{Pr} \beta_{2}=\beta_{2}^{\prime}\right]=(1 / 2)+(1 / 2) \varepsilon_{2}
$$

Here $\operatorname{Pr}\left[\beta_{2}=0\right]=\operatorname{Pr}\left[\beta_{2}=1\right]=1 / 2$. Because $\varepsilon_{2}$ is non-negligible, this shows that $B_{2}$ violates the assumption that the DDH is hard.

Theorem 3: The SEMU is semantically secure against a chosen keyword attack in the Game1 under the BDH assumption and a chosen plaintext attack in the Game 2 under the DDH assumption.

Proof: The theorem 3 is proved directly by theorem 1 and theorem 2. It shows that an IND-CPA adversary $A$ on the SEMU with the advantage $\varepsilon=\varepsilon_{1}^{\prime}+\varepsilon_{2}^{\prime} \quad$ as required.

Claim 1. If the EnKeyword() and the EnData(.) are secure encryption algorithms, then the SEMU can prevent revoked users from searching over encrypted data and accessing the data, thus achieving revocability.

Proof: A revoked user $u_{j}$ will no longer be able to search over encrypted data because the revoked user $u_{j}$ is removed from the ciphertext $B$. In the SEMU, when the cloud server processes the search queries of a user, it first obtains $B_{i}$ from $B\left(B=\sum_{i \in U} B_{i} N_{i} N_{i}^{\prime} \bmod N\right)$, and verified whether $B_{i}=H_{2}(\hat{e}(T, A))$. Where

$$
B_{i}=H_{2}\left(\hat{e}\left(p k_{i}^{r}, H_{1}(w)\right)\right), T=H_{1}(w)^{s k_{i}}, A=g^{r}
$$

If the user $u_{j}$ is revoked from the system, his corresponding $B_{j}$ should be removed from $B$. Without $B_{j}$, performing a search is impossible. Therefore, the revoked user $u_{j}$ cannot search over encrypted data and access the data.

Claim 2. The SEMU can control queries.

Proof: In the SEMU, the private key of an authorized user is needed to generate a valid trapdoor, and anyone cannot obtain such private key except for the authorized user himself. Without the private key of the authorized user, no one can generate a valid trapdoor, and the cloud server or outside attackers cannot perform trapdoor test processing. Therefore, the SEMU can control queries. 
Claim 3. The SEMU can provide query privacy.

Proof: The notion of query privacy in this paper is that secret information or data of authorized users may be leaked to an unauthorized party in the query process. In the SEMU, the cloud server or outside attackers cannot obtain any information about the keyword according to the trapdoor $T_{w}$ because of the secure one-way hash function and the difficulty in computing discrete logarithms. Moreover, the cloud server or outside attackers cannot obtain any information from the ciphertexts $C_{1}$ and $C_{2}$. Although the anonymous information $T_{3}=p_{i}$ of an authorized user is inevitably leaked to the cloud server, the cloud server cannot determine who corresponds to $p_{i}$, such that this information leakage to the cloud server is tolerable because no secret information is disclosed to the cloud server or to other users in the query phase. Therefore, the SEMU can provide query privacy.

Claim 4. The SEMU can achieve designated tester.

Proof: In the trapdoor test phase, the private key of the cloud server is needed to compute for test parameter $T$. Thus, outside attackers cannot perform trapdoor test processing without this private key. Therefore, only the designated cloud server can perform trapdoor test processing.

Claim 5.The SEMU can provide data confidentiality.

Proof: In the SEMU, data are encrypted by the data owner and then uploaded to the cloud server. Authorized users can recover the data using their private key. Both unauthorized users and the cloud server cannot recover the data because they do not possess the private keys of authorized users. Therefore, the SEMU can protect the data from both unauthorized users and the cloud server and provide data confidentiality.

Claim 6. The SEMU can provide query unforgeability.

Proof: The proof is straightforward. In the SEMU, the private key of an authorized user is used to generate valid keyword queries, such that neither other authorized users nor the cloud server can generate valid keyword queries on behalf of the authorized user unless the private key of this authorized user is disclosed. Therefore, the SEMU can provide query unforgeability.

\subsection{Performance}

In this section, we compare the performance of the SEMU with that of previous works.

We first view the ciphertext size of an encrypted keyword for $m$ users. In scheme [6], the ciphertext size of a encrypted keyword is $(m+3) L$, where $L$ is an element in $G$, and $m$ is the number of users. In schemes [20,21], the process of encrypting a keyword includes two parts: Enroll and GenIndex. The total ciphertext size in the two parts is $(m+1) L+\bar{L}$, where $\bar{L}$ denotes an element in $G_{T}$. However the total ciphertext size of the SEMU is $L+|N|$, where $|N|$ denotes $N$ size, and $N=p_{1} p_{2} \ldots p_{m}$. We use the fact that the prime order $p$ of $G$ is sufficiently large to guarantee that the discrete logarithm problem in $G$ is intractable. However, in the Chinese Remainder Theorem, $\left(p_{1}, p_{2}, \ldots, p_{m}\right)$ are positive integers that are relatively prime in pairs, so that $p>p_{m}>\ldots>p_{1}$. In other words, $(m+3) L>(L+|N|)$. Therefore, the ciphertext size of an encrypted keyword in the SEMU is shorter than that of the Schemes $[6,20,21]$ in a multi-user setting. Notably, short ciphertext size is efficient for the storage of the cloud server because the server should store a large amount of ciphertexts for a number of users.

If we view the number of transmitting components between the data owner and the authorized users or the data owner and the cloud server as communication cost, no additional traffic is generated in the SEMU compared with the previous schemes $[7,9,10$, 
11] in a single-user setting because the same number of ciphertexts are transmitted, although the content of packets are changed.

From the perspective of computational cost at an authorized user side, the SEMU mainly involves three exponentiation operations in generating the trapdoor phase. This computational cost is the same as that of schemes $[7,9,10,11]$ in a single-user setting and that of scheme [6] in a multi-user setting. In the decryption phase, the SEMU needs to perform one inverse computation, one exponentiation, and a multiplication operation, after which it performs one Exclusive or $(X O R)$ operation. This computational cost is almost the same as that of previous schemes $[7,9,10,11]$ in a single-user setting.

In scheme [6], when a user wants to send the same message with keyword $w$ to $m$ users, the sender should encrypt the keyword $w$ for $m$ users by additionally computing $B_{j}$ for $1 \leq j \leq m$ and the encryption of the message. When the cloud server searches the encryption with the keyword $w$, which a user $u_{i}$ wants to search, using the trapdoor of $u_{i}$, the cloud server has to take $O(m)$ time to test trapdoor matching with $\left(B_{1}, B_{2}, \ldots B_{m}\right)$. In schemes $[20,21]$, upon receipt of a query trapdoor for a keyword w from the user $u_{j}$, the server first looks for the helper key $h k_{u_{j}}$ of user $u_{j}$ in $\left(h k_{u_{1}}, h k_{u_{2}}, \ldots, h k_{u_{m}}\right)$ and then searches for the corresponding encryption with the keyword w by the $h k_{u_{j}}$. This process should take $O(m)$ time. However, in the case of the SEMU, the sender only needs to send $\mathrm{B}$, which is computed by the Chinese Remainder Theorem, to the cloud server, rather than sending $\left(B_{1}, B_{2}, \ldots B_{m}\right)$. Thus, the cloud server only needs to take $O(1)$ time to test the trapdoor matching with $B_{i}=B \bmod p_{i}$, which is the same as that in a single-user setting $[7,9,10,11]$. Therefore the SEMU is more efficient than schemes [6, $20,21]$ in terms of the query time at the server side. Notably, less query time is important for both user and server.

The SEMU has very efficient overhead for the storage of both the server and users compared with schemes $[6,20,21]$ in a multi-user setting. The SEMU requires only $n|C|+n(L+|N|)$ storage of a server for $\mathrm{n}$ data of $\mathrm{m}$ users, whereas scheme [6] and schemes $[13,14]$ respectively need $n|C|+n(m+3) L$ and $n|C|+n\left(m L+2\left|Z_{p}^{*}\right|\right)$ storage, where $C=\operatorname{EnData}\left(p k_{1}, p k_{2}, \ldots, p k_{m}, M\right)$. At the user side, the SEMU only needs to keep one private key $s k_{i}$, which is the same as that of schemes $[7,9,10,11]$ in a single-user setting and the scheme [6] in a multi-user setting, but fewer than that schemes $[20,21]$ in a multi-user setting.

More importantly, the SEMU does not rely on shared keys to generate valid encrypted keyword queries. In the SEMU, each authorized user has only one private key, which is different from that of other users. Using their own private key, authorized users can generate valid encrypted keyword queries and decrypt the encrypted data without knowing the keys of other users. This condition significantly simplifies user revocation, and is more efficient and practical for a multi-user setting. Although each authorized user in schemes [20,21] has a distinct query key to construct search queries, all authorized users share the same key to generate the index for the keyword w. which causes a weakness in that a revoked user can write records to the dataset. In addition, their schemes need to store a U-Hkey (containing user identities and their helper keys) on the cloud server, thereby increasing storage overhead on the cloud server. Furthermore, schemes $[20,21]$ use the fact that user revocation mainly depends on whether the cloud server removes the help key of the user from the U-Hkeylist. Although we assume that the cloud service provider is honest-but-curious, the cloud service provider may not remove the help key $h k_{u}$ of the user from the U-Hkey list when he can obtain some benefits. 
Table 1. Comparison of Previous Schemes with the Proposed SEMU

\begin{tabular}{lcccc}
\hline & {$[10]$} & {$[6]$} & {$[20,21]$} & SEMU \\
\hline Setting & single-user & multi-users & multi-users & multi-users \\
Designated Test & yes & no & no & yes \\
Controlling queries & yes & yes & yes & yes \\
query privacy & yes & yes & yes & yes \\
Revocation & no & no & incompletion & yes \\
Addition & no & no & yes & yes \\
\hline Ciphertext size & $L+\lambda+|\bar{C}|$ & $(m+3) L+|C|$ & $(m+1) L+|C|+\bar{L}$ & $L+|N|+|C|$ \\
& & & & \\
Trapdoor cost & $3 \exp$ & $3 \exp$ & $\exp$ & $3 \exp$ \\
Test cost & $\exp +\mathrm{BM}$ & $(m+2) \mathrm{BM}$ & $h_{k}+\mathrm{BM}$ & $\exp +\mathrm{BM}+\operatorname{Mod}$ \\
& & & & \\
User storage cost & $\left|Z_{p}^{*}\right|$ & $\left|Z_{p}^{*}\right|$ & $2\left|Z_{p}^{*}\right|$ & $\left|Z_{p}^{*}\right|$ \\
Server storage cost & $G+\lambda+|\bar{C}|$ & $(m+3) L+|C|$ & $(m+1) L+|C|+\bar{L}$ & $L+|N|+|C|$
\end{tabular}

$L$ : element in $\mathrm{G} ; \bar{L}$ : element in $G_{T}$; exp: exponentials;

$h_{k}$ : a keyed hash fuction; $\mathrm{BM}:$ bilinear pairings;

Mod: modulo $p_{i} ; m$ : the number of the authorized users;

$|N|: N$ 's size, where $N=p_{1} p_{2} \ldots p_{m}$;

$|C|: C$ 's size, where $C=\operatorname{EnData}\left(p k_{1}, p k_{2}, \ldots, p k_{m}, M\right)$;

$|\bar{C}|: \bar{C}$ 's size, where $\bar{C}=\operatorname{EnData}(p k, M)$.

Moreover, when some users are added or removed, the SEMU performs scalable encryption at the cloud server side without shipping the outsourced data back to the data owner,thus reducing communication overhead. When the decrypted key is set up and updated, the SEMU scheme does not require any interaction between the data owner and the authorized users.

The aforementioned analysis shows that the SEMU minimizes the communication and storage overhead for both the server and users as well as has efficient computation overhead compared with previous schemes in a multi-user setting. The SEMU achieves features similar to that of most existing schemes in a single-user setting and does not downgrade because of supporting multiple users. Table 1 shows a comparison between other schemes and the proposed SEMU in terms of performance and features. In this work, we omit small computation operations such as multiplication in $G$ or $G_{T}$.

\section{Conclusion}

In this paper, we have presented a searchable encryption scheme in a multi-user setting for cloud storage. When a user wants to share the same data with particular users, the proposed SEMU can be efficiently used for this application. The proposed SEMU avoids repeatedly encrypting the same data and the same keywords with the public keys of particular users using a general PEKS, thus reducing communication and storage overheads. The proposed SEMU performs scalable encryption on the cloud server without shipping the outsourced data back to the data owner when group membership is changed. We also have analyzed security and showed that the proposed SEMU is secure. For future work, we plan to extend our ideas for more types of queries. 


\section{Acknowledgments}

This work was partially supported by National Natural Science Foundation of China under Grants 61272415, 61070164; Natural Science Foundation of Guangdong Province, China, under Grant S2012010008767; Science and Technology Planning Project of Guangdong Province, China, under Grant 2013B010401015. This work was also supported by the Zhuhai Top Discipline -Information Security.

\section{References}

[1] D.Song, and D. Wagner, and A.Perrig, "Practical Techniques for Searching on Encrypted Data", IEEE Symposium on Research in Security and Privacy, Berkeley, CA, (2000), pp. 44-55.

[2] Z. Yang, S. Zhong, and R.N.Wright,"Privacy-preserving queries on encrypted data", Proc. of the 11th European conference on Research in Computer Security, (2006); Hamburg, Germany.

[3] M. Abdalla, M. Bellare, D. Catalano, E. Kiltz, T. Kohno, J.M. Lee, G. Neven, and H. Shi, "Searhable Encryption Revisited: Consistency Properties, Relation to Anonymous IBE, and Extensions", The 25th Annual International Cryptology Conference, Santa Barbara, (2005); California, USA.

[4] Y.C. Chang, and M. Mitzenmacher, "Privacy Preserving Keyword Searches on Remote Encrypted Data, "Applied Cryptography and Network Security, Third International Conference, ACNS, (2005); New York, USA.

[5] R. Curtmola, J. Garay, S. Kamara and R. Ostrovsky, "Searchable Symmetric Encryption: Improved Definitions and Efficient Constructions", Proceedings of the 13th ACM Conference on Computer and Communications Security, (2006); Alexandria, USA.

[6] Y. H. Hwang, and P. J. Lee, "Public Key Encryption with Conjunctive Keyword Search and Its Extension to a Multiuser System". Lecture Notes in Computer Science, vol. 4575, (2007), pp. 2-22.

[7] D. Boneh, G. di Crescenzo, R. Ostrovsky and G.Persiano, "Public key encryption with keyword search", Eurocrypt'04, (2004); Interlaken, Switzerland.

[8] J. Baek, R. Safavi-Naini, and W. Susilo, "Public key encryption with keyword search revisited". Proceedings of Applied Cryptography and Information Security, (2006); Glasgow, UK.

[9] M. Bellare, A. Boldyreva, and A. O'Neill, "Deterministic and efficiently searchable encryption", Lecture Notes in Computer Science, vol. 4622, (2007), pp. 535-552.

[10] H. S.Rhee, J. H.Park, W.Susilo, and D. H.Lee, "Trapdoor security in a searchable public-key encryption scheme with a designated tester", The Journal of Systems and Software, vol.837, (2010), pp.63-771.

[11] B. Joonsang, S. N. Reihaneh, S. Willy, "Public key encryption with keyword search revisited", Lecture Notes in Computer Science, vol. 5072, (2008), pp. 1249--1259.

[12] J. Ni, Y. Yu, Q. Xia, and L. Niu, "Cryptanalysis of Two Searchable Public Key Encryption Schemes with a Designated Tester", Journal of Information and Computational Science, vol.16, no.9, (2012), pp. 4819-4825.

[13] M. Nishioka, "Perfect keyword privacy in PEKS systems", Lecture Notes in Computer Science, Vol.7496, (2012), pp.175-192.

[14] C.Hu, and P.Liu, "An enhanced searchable public key encryption scheme with a designated tester and its extensions", Journal of Computers, vol.7, no.3, (2012), pp.716-723.

[15] P. Xu, H. Jin, Q.H. Wu, and W. Wang, "Public-Key Encryption with Fuzzy Keyword Search: A Provably Secure Scheme under Keyword Guessing Attack", IEEE Transactions on Computers, vol.61, no.11, (2013), pp. 2266-2277.

[16] Q. Liu, G. Wang, and J. Wu, "Efficient Privacy Preserving Keyword Search Scheme in Cloud Computing", The 12th IEEE International Conference on Computational Science and Engineering, (2009); Vancouver, Canada.

[17] Q. Liu, G. Wang, and J. Wu, "Secure and privacy preserving keyword searching for cloud storage services", Journal of Networks and Computer Applications, vol.35, no.3, (2012), pp. 927-933.

[18] L.Chen, and Q.Y.Wen,"A novel privacy preserving keyword searching for cloud storage", 2013 Eleventh Annual Conference on Privacy, Security and Trust (PST), Tarragona, (2013).

[19] R. Curtmola, J.Garay, S. Kamara, and R.Ostrovskey,"Searchable Symmetric Encryption: Improved Definitions and Efficient Constructions", Proceedings of ACM Conference on Computer and Communications Security, (2006); Alexandria, USA.

[20] F.Bao, R.H.Deng, X.Ding, and Y.Yang, "Private Query on Encrypted Data in Multi-user Settings", Information Security Practice and Experience, 4th International Conference, (2008); Sydney, Australia.

[21] Y.Yang, X.Ding, R.H.Deng, and F.Bao, "Multi-User Private Queries over Encrypted Databases", International Journal of Applied Cryptography Archive, vol.1, no.4, (2009), pp. 309-319.

[22] M.Abdalla, M.Bellare, and P.Rogaway, "DHIES: an encryption scheme based on the Diffie-Hellman problem", Lecture Notes in Computer Science, vol. 2020, (2001), pp. 143-158.

[23] D.Boneh, and M.Franklin, "Identity Based Encryption from the Weil Pairing", SIAM Journal of Computing, vol.32, no.3, (2003), pp.586-615. 
International Journal of Security and Its Applications

Vol. 11, No. 7 (2017)

[24] Y.D. Luuu, M.L. Wu, "A fully public key traitor tracing scheme. WSEA Transaction on Circuits", vol.1, (2002), pp.88-93 